IX.

DER

\title{
NERVÖSE APPARAT
}

IN DEN

ENDSCHEIBEN DER FROSCHZUNGE.

VON

DR. MED. J. NIEMACK.

Aus dem anatomischen Institut in Göttingen.

Mit Fig. 6 und 7 auf Tafel XIIIXIII. 

Im Anschluss an meine Beobachtungen über den akustischen Endapparat untersuchte ich mit der gleichen Methode auch noch die sog. Nervenendscheiben auf der Froschzunge und gestatte mir, über sie ebenfalls einige Mitteilungen zu machen. Ehrlich hatte schon bei der Publikation $\left.{ }^{1}\right)$ seiner Entdeckung über das bequeme Objekt eine kurze, den Zuhörern seines Vortrages durch eine Tafel veranschaulichte Erörterung gegeben. Den Nervenreichtum dieser Scheiben schildert er so gross, dass dieselben bereits makroskopisch durch intensiv blaue Farbe hervortreten. Dicht unter dem Epithel sei ein dichtester Plexus mit mehr oder weniger grossen Varikositäten. An letztere legten sich die Sinneszellen mit ihren Enden an, ohne doch mit ihnen zu verschmelzen. Aus diesem Grundplexus träten weiterhin in's Epithel feine Stämmchen über, von denen ein Teil sich zu den Sinneszellen begebe, um an deren Oberfläche mit einem höchst scharfen kleinen Knöpfchen zu endigen. So seien die Nerven mit ihren zugehörigen Zellen nicht per continuitatem, sondern per contiguitatem verbunden. Arnste in ${ }^{2}$ ) hatte diese Bemerkungen im wesentlichen bestätigen können. Er fand ferner, dass aus dem dichten Nervenplexus sich netzartig angeordnete variköse Fäden entwickeln, die bis ans Epithel reichen. Von da aus treten feinste Fäden bis an die Oberfläche. Die Epithelzellen bleiben ungefärbt bis auf Gebilde, welche Arnstein für die Axel-Key'schen Geschmacks-

1) Deutsch. Mediz. Wochenschr. 1886.

2) Anat. Anz. II. S. 1251887.

Anatomische Hefte I. Abteilung V. Heft. 
zellen hält. Ihr Körper nebst Kern seien gefärbt, der centrale dagegen, sowie der periphere Fortsatz seien ungefärbt geblieben. (?) Ausser diesen fand er noch keulenförmige Zellen mit langem, in eine Nervenfibrille übergehenden centralen Fortsatz. Endlich bemerkt Arnstein, dass auch Merkel's „Flügelzellen“ sich färbten. Nach ihm versuchte sich Fajerstajn ${ }^{1}$ ) an derselben Aufgabe. Derselbe giebt zuerst seine nach anderen Methoden gewonnenen Resultate über die einzelnen Zellengebilde der Endscheibe, wobei er den von $\mathrm{Merkel}^{2}$ ) als Kunstprodukte beseitigten Gabelzellen wieder zur Existenz zu verhelfen, und die "Stäbchen“(Sinnes-)Zellen als mit den Stützzellen identische Gebilde zu erweisen bemüht ist. Es ist nicht unsere Absicht, auf die histologischen Details hier weiter einzugehen. Sie werden später noch gestreift werden müssen. Wir wenden uns daher gleich zu den nach Ehrlich ausgeführten Untersuchungen, mit deren Mitteilung Fajerstajn seine Arbeit abschliesst. Fajerstajn hat entweder in die Bauchvene injiziert, oder auch den Farbstoff direkt in die Mundhöhle des Tieres gegossen. Auffallend ist, dass Fajerstajn noch zwei Jahre nach Veröffentlichung des Fixationsverfahrens von Smirnow ${ }^{3}$ ) nur mit Quetschpräparaten und ohne Fixation gearbeitet hat, obwohl ihm die Arnstein'sche Publikation bekannt ist. Seine Angaben sind in Kürze folgende: Die in der Höhe dẹ Kapillarschlinge ihr Mark verlierenden Nerven bilden einen varikösen „Plexus subbasalis"; von diesem treten nun feinere variköse Fädchen ab, um einen „Plexus subepithelialis" zu bilden, welcher in derselben Höhe liege, wie das von den feinsten Fortsätzen der Flügel- u. a. Zellen gebildete basale Netzwerk. Von da aus gehen neue Fasern entweder bis

1) Recherches sur les terminaisons des nerfs dans les disques terminaux chez la grenouille. Varsovie. Arch. de Zool. expér. et gén. sér. Ir t. VII. 705. 1889 mit 'Tafel.

2) Merkel, Über die Endigungen in der Haut d. Wirbelt. Rostock 1880.

3) Arnstein l. $c$. 
zur Oberfläche, oder in irgend eine beliebige Höhe des Epithels. Sie enden sämtlich mit mehr oder weniger grossen Endknöpfchen („Renflements"). Da bei Ehrlich's Methode „ni les corps des cellules ni leurs noyaux n'y sont point visibiles!" so sei es unmöglich, den Ort ihrer Endigung genau zu präzisieren ${ }^{1}$ ). Einige Fasern beschreiben im Epithel einen Bogen, dessen Gipfel oft weit höher liegt, als sein Ende. Die grossen vorkommenden „Renflements" könne man zunächst versucht sein für Sch wann'sche Kerne zu halten, allein die färben sich nicht. (?) Zweierlei Zusammenhaug zwischen Zelle und Nerv sei vorhanden: 1. die Endknöpfe legen sich an den Körper der Zelle an, 2. die Zellenfortsätze ruhen in dem von Nervenplexus und Zellfortsätzen gemeinsam gebildeten basalen Rasen. Hätte Merkel mit seiner Wertschätzung der Stäbchenzellen als ,Sinnes"-Zellen recht, so müsste den Anhäufungen dieser Zellen eine Anhäufung von Nervenfibrillen entsprechen, was nicht der Fall sei. Fajerstajn ist vielmehr geneigt, die "Gabelzellen" als eigentliche Sinneszellen zu bezeichnen, oder auch die ganze Endscheibe nur in toto als Organ der Sinnesentwickelung gelten zu lassen. Übrigens hält auch Fajerstajn die Endscheiben nicht für Geschmacks-, sondern für Tastorgane. Die von Arnstein beschriebenen keulenförmigen Zellen mit den langen fadenförmigen Fortsätzen seien ohne Verbindung mit dem Nerv, vielleicht seien es entzündliche, Wanderzellen"! Wir haben uns mit Fajerstajn's Untersuchung etwas eingehender beschäftigen müssen, weil dieselbe gewissermassen als eine Monographie über die Endscheiben der Froschzunge auftritt und verschiedene sehr frappierende Sätze enthält. Zu letzteren rechne ich die Vorstellung, dass ein Filzgeflecht aus Nerv und Zellfortsätzen als Endapparat fungiere, dass die "Gabelzelle" als Sinnesepithel aufgefasst und diese Eigenschaft den Stäbchenzellen bestritten wird, endlich

1) 1. c. 739 . 
dass eine bestimmte langgeschwänzte, keulenartige Zellenform als "Wanderzellen" gedeutet wird. Alle diese Dinge hat Fajerstajn nur an nicht fixierten Präparaten studiert.

Es liegt aber auf der Hand und wird auch von Fajerstajn ausdrücklich betont, dass bei der Flüchtigkeit und den übrigen oben erwähnten Eigenschaften der nach Ehrlich behandelten Präparate, in denen die einzelne ungefärbte Zelle kaum differenzierbar ist, nur nach Fixation und folgender Anfertigung von Zupf- oder Schnittpräparaten über das Verhältnis des Nervs zur Zelle Anschauung genommen werden kann. Daher könnten F ajerstajn's Angaben, dass zwischen den Stäbchenzellen und den Nerven keine Verbindung bestehe, nur dann Eindruck machen, wenn er über die Anfertigung unfixierter frischer Präparate hinausgegangen wäre. Eine sorgfältige Nachuntersuchung mit verbesserter Methode wird demnach nicht überfüssig sein.

Zur Methode sei bemerkt, dass auch für die Froschzunge sich die von mir verwandte dünne Farblösung bewährt hat. Man trennt mit der krummen Scheere ein beträchtliches Stück der Epithelschicht flach mit möglichst wenig Muskelfasern von der Zunge ab. Dasselbe darf etwas länger in der Farblösung verweilen, da es meist in die Tiefe untersinkt und dem Einfluss des Sauerstoffs nicht in gleicher Weise wie das Gehörorgan ausgesetzt ist. Aus demselben Grunde pflegt die stärkste Färbung des Nervengeflechts in den Endscheiben etwas langsamer einzutreten. Der Weg, auf welchem die Färbung fortschreitet, ist ein etwas eigentümlicher und nie an allen Scheiben gleichmässiger. Während der tiefste Plexus und die periphere Partie des zutretenden Nervenbündels schon gefärbt sind, sieht man auf der Scheibe kleinste blaue Pünktchen zunächst ohne Zusammenhang mit den Nerven. Unter Verschiebung des Tubus ist der Moment abzupassen, wo sich eine Kontinuität zwischen diesen Punkten und dem Nervengeflecht hergestellt hat. Dann wird fixiert. Trotzdem wird man nicht gerade häufig an ein er Scheibe alles haben. 
Ob Nichtgefärbtes an dieser Scheibe nicht vorhanden ist, sondern nur an einer anderen, oder ob es nur aus unbekannten Gründen der Färbung Schwierigkeit entgegensetzt, wird vielleicht in $\mathrm{Zu}$ kunft aufgeklärt. Versuchsweise habe ich wohl das Organ bis 24 Stunden in der Flüssigkeit gelassen, ehe ich es zur Untersuchung nahm. Abgesehen von der dann eingetretenen Färbung sehr verschiedenartiger Epithelzellen war in diesen Fällen von den feineren Nervenfasern nichts übrig geblieben als die beträchtlich aufgequollenen Varikositäten, fast ohne jede fibrilläre Verbindung. Zur Differenzierung der Zellen wird es sich möglicherweise empfehlen, die verschieden lange Dauer bis zur Färbung jeder einzelnen Zellengattung genauer zu studieren. Über diesen Gegenstand sei hier nur bemerkt, dass bei meinem Verfahren die „Flügelzellen“ Merkel's sich so gut wie niemals bläuen, dass dagegen ausser einigen hin und wiedẻr im gewöhnlichen Epithel der Zungenoberfläche vorkommenden anscheinend normalgestalteten Flimmerzellen die von A rns tein beschriebenen keulenförmigen Zellen mit äusserst langem feinem, bisweilen gespaltenem unteren Fortsatz sich immer intensiv färben. Ihre centralen fibrillenähnlichen, doch nie varikösen Fortsätze haben, wie ich bestimmt gegen Arnstein versichern kann, nichts mit Nerven gemein (Fig. 7 a). Ich habe nie einen Zusammenhang ihres Schwanzes mit dem Nervengeflecht gefunden. Häufig umgeben sie, in bestimmten Zwischenräumen in das periphere Flimmerepithel eingesprengt, kranzartig die ganze eigentliche Endscheibe. Doch noch häufiger liegen sie ganz unregelmässig zerstreut; aber immer nur in der Peripherie der Scheibe.

Die Stäbchen-, Stütz- u. dergl. Zellen nehmen innerhalb des angegebenen Zeitraumes keine Färbung an. Etwas später finden sich dagegen gefärbte Zellen von der Gestalt einer sehr langhalsigen, kurzbauchigen Flasche, der Kern mit stark tingiertem Nucleolus, die ich mit Arnstein für die „Geschmackszellen" halte, d. i. für die Sinneszellen, ohne damit über die 
Funktion der Zungenscheibe als Geschmacks- oder Tastorgan etwas aussagen zu wollen (Fig. $7 \mathrm{~b}$ ).

Es ist da keine völlige Konstanz vorhanden. Als ich an Sommerfröschen die im Winter erhaltenen Resultate kontrollierte, konnte ich in jedem Falle nur die Angabe Arnstein's bestätigen, dass Zellen, deren Gestalt durchaus den „Stäbchenzellen“ entsprechen, aber auch nur diese samt ihren Kernen sich färbten. Die Färbung des Plasma war grob granuliert und diese Granula schienen bei sorgfältiger Beobachtung gerade wie beim Gehörorgan der Zellenoberfläche allein anzugehören. Liess ich das Präparat 30 Minuten in der etwas stärkeren Farblösung, so geriet die Nervenfärbung am besten, nach 60 Minuten umgekehrt die der Zellen. Daher war nur sehr selten das Verhältnis des Nervenplexus zu diesen Zellen scharf sichtbar (Fig. 7 c).

Wir wenden uns zur eigentlichen Beschreibung des Nervenverlaufes. Unter den zur Papille hintretenden Nerven zeichnet sich in der Regel eine Faser durch etwas grössere Stärke aus. Nach Verlust der Markscheide, welcher in der Höhe der Kapillarschlinge zu erfolgen pflegt (Fajerstajn); teilen sich die freien Achsencylinder teils dichotomisch in gleichstarke Zweige, teils geben sie ein feineres Ästchen ab, während der eigentliche Stamm erst weiterhin zerfällt. Auch hier, wie beim Gehörorgan ist die Dichotomie Regel, doch nicht ausnahmslose (Fig. 6). Die so entstehenden feineren Fasern laufen ohne jede Varik osität, und nur an den Teilungsstellen dreieckig aufschwellend, mehr oder weniger geschlängelt nach der Peripherie hin auseinander. Unterwegs senden sie sich gelegentliche, doch im ganzen seltene Anastomosen zu. Ganz wie am akustischen Endapparat wird auf diese Art ein Netzgefleeht gebildet. Dasselbe liegt in dem subepithelialen Gewebe und hat noch keine Beziehungen zu Zellen.

Aus ihm lösen sich überall, besonders stark aber in den peripheren Regionen der Scheibe mehr oder weniger lange 
variköse Fasern. Die Anzahl der Anastomosen, welche diese letzteren sich zusenden, ist eine beschränkte. Oft verläuft eine solche variköse Faser eine beträchtliche Strecke weit dahin, ohne in Berührung mit anderen zu kommen. Gegen den Rand zu biegen dieselben sehr häufig in Gestalt einer nach dem Centrum zu offenen horizontalen Schlinge, welche gerade Raum für eine Zelle bietet, um (Fig. 6 a). So bildet sich mit ziemlicher Konstanz ein System von Randschlingen in der Höhe der untersten Kernschicht des Epithels, welches jedoch kein eigentliches Netz darstellt; zwischen je zwei Schlingen bleibt Raum für mehrere andere Zellen frei. Auf diesem ,Plexus subepithelialis" Fajerstajn's, der aber nicht eigentlich unter dem Epithel liegt, sondern dessen Lücken - wenigstens am Rande - bereits durch die Zellen bestimmt werden, stehen nun auch vertikale Schlingen. Oft treffen zwei oder drei Fasern in einer Varikosität zusammen, eine Zelle in Kernhöhe umgreifend. Stets handelt es sich dabei um Zellen der tieferen Regionen, nie um das die Oberfläche deckende, mit flachgestellten Kernen versehene Cylinderepithel. Die gelegentlich innerhalb der unteren Epithelschichte gesehenen, mit einer grösseren oder schwächeren Varikosität abschliessenden scheinbaren Nervenendigungen halte ich nach lange darauf gerichteter Aufmerksamkeit für mangelhafte Färbungsresultate. Je vollkommener die Färbung gelang, um so seltener waren sie vorhanden, um so sicherer konnte auf die Fortsetzung solcher, ,intraepithelialer freier Endigungen“ nach irgend einer Seite hin gerechnet werden. Es scheint mir, dass innerhalb des Epithels Nervenendigung zu den grössten Seltenheiten gehört, dass vielmehr dort jede Varikosität nach beiden Seiten hin mit einer anderen fibrillär verbunden ist.

Aus diesem Plexus erheben sich nun Endfibrillen, welche zwischen den Epithelzellen bis zur freien Oberfäche aufsteigen. Häufig treten mehrere Fäserchen in einer Varikosität zusammen, um gemeinsam eine Endfibrille zu bilden (Fig. 7d). In einer 
ganzen Reihe von Präparaten lag zwischen je zwei Cylinderzellen ein freies Nervenende. Dasselbe trägt eine mehr kolbenoder mehr knotenartige Verdickung, in manchen Partien erschien es förmlich granuliert; man wird auf solche besondere Formen jedoch wenig Gewicht legen dürfen, nachdem Feist (s. o.) es wahrscheinlich gemacht hat, dass es sich um Absterbeerscheinungen handelt. Es überragen diese freien Endknöpfchen das Epithel um ein Geringes. Ein genügender Schutz gegen allzu energische Einwirkung seitens des Mundhöhleninhaltes dürfte wohl durch den stets reichlich vorhandenen Schleim gegeben sein.

Neben dieser freien Endigung geht eine zweite Endigungsart scheinbar selbständig einher, welche durch Zellen vermittelt wird. An macerierten und zerzupften Präparaten wurden bisweilen die oben schon erwähnten „Stäbchenzellen“ gefunden von rauhem, grob granuliertem Aussehen (Fig: 7), an deren unterem Ende ein mit einer varikösen Anschwellung sich der Zelle anlegendes, kurzes Nervenendchen hạ̈ngen geblieben war. Dies geschah allerdings nicht häufig; vielmehr wurde regelmässig durch die Maceration der Zusammenhang zwischen Nerv und Zelle offenbar derart gelockert, dass man beim Zerzupfen beides für sich erhielt: die freien Zellen ohne irgend gefärbtes Anhängsel, die Nerven als ein leeres Maschenwerk mit Ausläufern und varikösen Fädchen. - Diese Bilder wurden auf's vorteilhafteste ergänzt durch jene Schnitt-Präparate, an denen ausser den. Nerven auch zugleich noch die "Stäbchenzellen", und nur diese, schön blau gefärbt waren. Überall waren ohne Mühe Verbindungsfädchen zwischen dem Nervengeflecht und diesen Zellen sichtbar. - Hiernach kann ich nicht daran zweifeln, dass die „Stäbchenzellen“, und sie allein, nervöser Natur sind, dass alle anderen Zellenarten dagegen wesentlich als Stützmaterial zu betrachten sind. Eine Kontinuität zwischen diesen StäbchenZellen und dem Nerven, auf welche die letzterwähnten Schnitt- 
präparate wohl hätten gedeutet werden können, ist doch durch die Ergebnisse der Macerationspräparate mit ziemlicher Sicherheit ausgeschlossen. Es ist durchaus dasselbe Verhältnis wie beim Ohr; und so bin ich geneigt auch hier dem blauen Mantel der Stäbchenzelle die Vermittlerrolle zwischen Zelle und Nerv zuzuschreiben.

Endlich sah ich gelegentlich in den letzterwähnten Präparaten Bilder, als ob eine dünne, aus dem Plexus aufsteigende Fibrille einen ungefärbten Zellenkern umgriffe und sich dann glatt bis zur Oberfläche fortsetze. Über das Verhalten des Plasmas konnte ich nichts wahrnehmen. Ob es sich hierbei um Resultate mangelhafter Färbung handeln kann, will ich nicht entscheiden; doch erscheint es beachtenswert, dass solche Bilder nur in den bestgeratenen Präparaten auftraten. Ihre Deutung (Kontinuität ?) bleibe einstweilen unentschieden (Fig. $7 \mathrm{e}$ ). Einzelne runde Zellen mit granuliertem Plasmamantel sind fast in jeder Endscheibe in der Tiefe aufzufinden; der Kern derselben bleibt ungefärbt ${ }^{1}$ ).

Unsere Beobachtungen gehen also dahin, dass, wie schon Ehrlich sah, keine Kontinuität, sondern nur Kontiguität zwischen Nerv und Zelle vorhanden ist und daneben noch eine freie Endigung auf der Oberfäche sich findet. Durch letztere kann die Bedeutung der Zellen für die Sinneswahrnehmung schon deswegen nicht beseitigt werden, weil die Verwendung einer so bedeutenden Nervenmasse, wie sie der Plexus bietet, zur Erzeugung der verhältnismässig geringfügigen freien Enden gar keine Analogien bieten würde. Wir haben aber auch oben einen Zusammenhang zwischen Stäbchenzellen und Nerv direkt beschrieben, und besitzen meines Erachtens in den Faserschleifen, welche je eine Zelle in der Gegend ihres Kerns umgreifen, einen

1) Auf die z. T. sehr interessanten Zellenformen ausserhalb der Endscheiben, welche sich nach Ehrlich's Methode färben, kann ich hier nicht eingehen. Eine derselben ist in Fig. $7 \mathrm{f}$. gezeichnet. 
direkten Hinweis darauf, dass diese verhä̉itnismässig wenigen einzelnen Zellen wie anatomisch, so auch physiologisch in näherer Beziehung zu nervösen Funktionen stehen. Ob dieses nur die Stäbchenzellen sind oder auch noch andere, lässt sich bisher nicht ganz bestimmt sagen. Die absolute Zahl gefärbter Stäbchenzellen war regelmässig geringer als die Anzahl der Netzmaschen. Es ist aber nicht sicher, dass diese Zellen sämtlich sich gefärbt hatten. Ein Siebplexus wie beim Ohr ist nicht vorhanden und wird der Schluss daher nicht unberechtigt sein, dass die Sinneszellen in der Zunge in verhältnismässig weit geringerer Zahl vorhanden seien als im Ohr. - Dem Umstand, dass ein grosser Teil der Nervenschleifen in etwa derselben Höhe liegt wie ein Teil der centralen Zellenfortsätze (Fajerstajn), werden wir nach unseren Allgemeinvorstellungen von Nerv und Zelle eine fundamentale, funktionelle Bedeutung nicht leicht beimessen.

An der Zunge tritt der Mangel der Ehrlich'schen Färbung, dass zwar gewisse Dinge (Nerven) sich stets gleichmässig, andere (Endzellen) aber sehr ungleich färben, besonders deutlich hervor und hindert noch, ein definitives Urteil auszusprechen.

Was die Funktion der Endscheibe betrifft, so möchte es doch nicht auszuschliessen sein, dass ihrer doppelten Nervenendigung auch eine doppelte Funktion entspricht. 